\title{
DIVERSIDADE DE QUINZE ESPÉCIES DE BORBOLETAS (LEPIDOPTERA, PAPILIONIDAE) EM SETE COMUNIDADES DE SANTA MARIA, RS.
}

\author{
DIVERSITY OF FIFTEEN SPECIES OF BUTTERFLIES (LEPIDOPTERA, PAPILIONIDAE) \\ IN SEVEN COMMUNITIES OF SANTA MARIA, RS, BRAZIL.
}

\section{Gustavo Schwartz ${ }^{1}$ Rocco Alfredo Di Mare ${ }^{2}$}

RESUMO

\begin{abstract}
O trabalho compara a diversidade estimada para sete áreas de Santa Maria, RS, com diferentes níveis de antropização, com base na abundância, similaridade e constância de 15 espécies de papilionídeos. O estudo foi realizado de Setembro de 1994 a Março de 1995, e de Novembro de 1995 a Fevereiro de 1996. As capturas foram efetuadas em intervalos médios de trinta dias, utilizando rede entomológica convencional, durante intervalos médios de 4 horas. As borboletas foram transportadas em envelopes glicinados, individualizados e etiquetados, para o laboratório do Departamento de Biologia da UFSM. Os dados sugerem que a cobertura vegetal desempenha uma função importante na distribuição e abundância dessas borboletas, e os mecanismos que agem em escalas geográficas mais amplas podem ser diferentes daqueles que atuam em escalas menores. Habitats, como capões e ambientes urbanos, representam locais favoráveis onde as borboletas podem usar esses ambientes complexos, exibindo uma norma de reação que minimiza a competição e a ação de inimigos naturais.
\end{abstract}

Palavras-chave: Insecta, Rhopalocera, Papilionoidea .

\section{SUMMARY}

The research has as objective to compare the esteemed diversity for seven of Santa Maria's counties, RS, Brazil, with different urbane levels, based on the study of abundance, similarity and steadiness among 15 Papilionidae species, from September 1994 to March 1995 and November 1995 to February

\begin{abstract}
1996. Captures were performed in intervals of thirty days, using entomological net, during intervals of 4 hours average. The butterflies were transported in glassine envelopes, individualized, properly labeled to the Laboratory at UFSM Biology Department. The results suggest that the canopy carries out an important function in the distribution and abundance of those butterflies. The mechanisms that act in wider geographical scales can be different from those that act in smaller scales. Coppices and urban landscapes can represent locals, where butterflies can use these complex environments exhibiting a reaction norm that minimizes competition and natural enemies' actions use these complex environments exhibiting a reaction norm that minimizes competition and natural enemies' actions.
\end{abstract}

Key words: Insecta, Rhopalocera, Papilionoidea.

\section{INTRODUÇÃO}

A distribuição de espécies, dentro de uma determinada área geográfica, é irregular em diferentes graus devido às características físicas, abundância de recursos e inimigos naturais. Geralmente, espécies que usam diferentes recursos podem atingir altas densidades, colonizando diversos locais em uma área relativamente grande. Por outro lado, espécies que utilizam recursos restritos não são abundantes, restringindo sua distribuição a locais específicos de uma área geográfica (BROWN, 1984).

\footnotetext{
${ }^{1}$ Aluno do Curso de Ciências Biológicas do Centro de Ciências Naturais e Exatas (CCNE),Universidade Federal de Santa Maria (UFSM).

${ }^{2}$ Farmacêutico Bioquímico-Professor Asssistente do Departamento de Biologia, CCNE, UFSM. Campus Universitário, Faixa de Camobi - km 9. Santa Maria, RS, Brasil.97105-900. E-mail: ram13@ zaz.com.br. Autor para correspondência.

Recebido para publicação em 23.09.98 Aprovado em 24.05.00
} 
Padrões na diversidade de insetos ilustram o papel estrutural das plantas ao criarem uma heterogeneidade ambiental, que sustenta uma alta diversidade entre organismos que dependem das plantas. A heterogeneidade das fontes alimentares pode providenciar uma explicação alternativa para um fenômeno mais consistente em ecologia, a distribuição e a abundância de espécies. A distribuição é ubíqua na natureza e pode ser produzida por uma variedade de processos, interpretada geralmente em termos de partição de nicho, forma que as espécies dividem os recursos em um contexto ecológico (competição) e evolutivo (substituição de caracteres e espécies). Entretanto, se as espécies não estão em competição, podem estar representando tipos funcionais diferentes, utilizando diferentemente o ambiente (SUGIHARA, 1980). A distribuição da abundância de espécies pode ser o resultado das propriedades do ambiente em si, em lugar das propriedades das espécies (HUSTON, 1995).

Em uma mesma área geográfica, a diversidade pode ser analisada dentro e entre habitats diferentes, refletindo a coexistência entre organismos, interagindo através da competição, por utilizarem as mesmas fontes ou o mesmo ambiente (WHITTAKER, 1967). As interações competitivas podem levar à exclusão competitiva e forte seleção natural para caracteres que produzem diferenciação de nicho e, provavelmente, as duas conseqüências primárias consistentes com a teoria do equilíbrio competitivo: a) diversidade total de espécies relativamente baixa; b) coexistência de espécies suficientemente diferentes para evitar a competição por fontes críticas (HUSTON, 1995).

O padrão espacial da diversidade entre diferentes locais pode incorporar os conceitos de diversidade $\alpha$ e $\beta$. A diversidade $\alpha$ representando o número de espécies em uma determinada área, e a $\beta$ o modo como os organismos respondem à heterogeneidade ambiental. A $\beta$ é expressa pelo índice de similaridade entre comunidades de diferentes habitats, ao longo de um gradiente, em uma mesma área geográfica. A $\beta$ resulta da baixa similaridade entre a composição de espécies de diferentes habitats ou localidades (WHITTAKER, 1960; WILSON \& MOHLER, 1983).

A família Papilionidae forma, possivelmente, um grupo monfilético com as famílias Pieridae $\boldsymbol{e}$ Nymphalidae (ACKERY \& VANE-WRIGHT, 1984), representada na região neotropical por cerca de 120 espécies, ocupando uma ampla gama de habitats (HEPNNER, 1991). Em Santa Maria, ocorrem 15 espécies, distribuídas de modo particular, desde a floresta decidual e semidecidual, até locais completamente urbanizados. O estudo da diversidade e estrutura das suas populações, em ambientes naturais e perturbados, são importantes por razões práticas, no que tange à conservação de borboletas e seus ambientes. Essa família constitui um grupo taxonômico bastante conhecido, podendo servir como um representante da biota ao seu redor.

O presente trabalho tem como objetivo comparar a diversidade em sete comunidades de Santa Maria, RS, com diferentes níveis de antropização, com a abundância, similaridade e constância de 15 espécies de papilionídeos.

\section{MATERIAL E MÉTODOS}

\section{Locais estudados e amostragem}

Os locais estudados situam-se entre os paralelos $53^{\circ} 10^{\prime}$ e $54^{\circ} 40^{\prime} \mathbf{W}$ e entre $29^{\circ} 00^{\prime}$ e $29^{\circ} 20^{\prime} \mathbf{S}$. Rincão do Canto (RC), Vale do Diabo (VD), Rincão do Soturno (RS), Morro do Link (ML) e Água Negra (AN) estão em áreas remanescentes da floresta decidual e semi-decidual da Serra Geral, com altitude inferior a $500 \mathrm{~m}$. A mata é formada por, aproximadamente, 200 espécies arbóreas e 135 são exclusivas (KLEIN, 1983). A geomorfologia é da formação da Serra Geral do Cretáceo, formada por diversos derrames basálticos, intercalados por arenito, caracterizada fisionomicamente por vales e montanhas. Cinco extratos vegetais são observados: emergente; arbóreo contínuo; arvoretas; arbustivo e herbáceo. As áreas de Schöenstatt (SC) e Camobi (CB), eminentemente urbanas, apresentam uma flora caracterizada por plantas ornamentais cultivadas, como: Verbenaceae, Malvaceae, Asteraceae, Mirtaceae e Apocinaceae. Schöenstatt apresenta grande diversidade de flores e Camobi com poucas, porém abundantes em número.

As observações foram realizadas entre setembro de 1994 a março de 1995 e entre novembro de 1995 a fevereiro de 1996, em intervalos médios de trinta dias para cada localidade estudada, utilizando rede entomológica convencional, durante intervalos médios de 4 horas. Os imagos foram acondicionados em envelopes glicinados, individualizados, devidamente etiquetados e transportados 
para o Laboratório do Departamento de Biologia da UFSM. Em AN, RS, RC e VD, as coletas foram realizadas em trilhas e estradas existentes, previamente escolhidas. Em $\mathbf{C B}$, eram percorridas ruas do bairro, escolhidas pelo número de casas com jardins apresentando flores. Em SC, foi investigada uma mata secundária, no Seminário de Schöenstatt. As espécies foram identificadas a partir de TYLER $\boldsymbol{e} \boldsymbol{t}$ al. (1994).

\section{Parâmetros estudados e análise dos dados}

A análise das áreas estudadas foi realizada através:a) da proporção da cobertura vegetal, a partir de fotografias aéreas, através do programa SITER (Sistema de Informações Territoriais Rurais, versão 1.0), gentilmente cedido pelo Departamento de Engenharia Rural da UFSM; b) dos índices de Shannon-Wiener $\left(\mathbf{H}^{\prime}\right)=-\Sigma$ pi $\mathbf{l n}$ pi e de Simpson $(\mathbf{S})=\Sigma(\mathbf{p i})^{2}$; onde pi representa a freqüência relativa de cada espécie; c) do índice de similaridade, calculado pelo método proposto por WARD (1963), com o programa "Statistica for Windows" versão 2.0. Esse método utiliza uma análise da variância para analisar a distância entre os grupamentos, minimizando a soma dos quadrados de quaisquer dois grupos hipotéticos formados a cada passo. No agrupamento das áreas, foi utilizada a Correlação de Pearson. A distância utilizada foi a Distância Euclidiana $\left(=\left\{\Sigma(\mathbf{x}-\mathbf{y})^{2}\right\}^{1 / 2}\right) ;$ d) do Índice de Constância: expressa a percentagem de espécies presentes nos levantamentos efetuados, calculado através da fórmula: $\mathbf{C}=\mathbf{p} \times \mathbf{1 0 0} / \mathbf{N}(\mathbf{p}=$ coletas contendo a espécie estudada e $\mathbf{N}=$ total de coletas), resultando as seguintes categorias: a) constantes $(\mathbf{C})$, presentes em mais de $50 \%$ das coletas; b) acessórias (A), presentes em $25 \%-50 \%$ das coletas e c) acidentais (Ac), presentes em menos de $25 \%$ das coletas (BODENHEIMER, 1955); e) da Equitabilidade: distribuição de indivíduos entre as espécies (POOLE, 1974). Corresponde à razão entre H' (índice de Shannon-Wiener para a amostra) e H'máximo (diversidade máxima); f) da Curva de Importância (POOLE, 1974), que expressa a riqueza e uniformidade na distribuição das espécies, em que a freqüência de cada espécie da comunidade é organizada em ordem decrescente de abundância e enumerada em ordem crescente; $g$ ) do teste $\mathrm{t}$ de Student, comparando os índices de diversidade $\mathbf{H}^{\prime}$ entre as áreas (POOLE, 1974).

\section{RESULTADOS E DISCUSSÃO}

Os resultados mostram a ocorrência de um gradiente na proporção de mata, formando dois grupos principais (Figura 1): RC, VD, RS e ML num grupamento, onde as duas primeiras apresentam cerca de $67 \%$ da área coberta por mata e as duas últimas com cerca de 50 e, fechando o cluster, está $\mathbf{A N}$, com uma cobertura formada por cerca de $1 / 3$ de mata, restrita a capões. O outro grupo é formado por SC e CB. O fenograma sugere haver um gradiente associado com o grau de antropização dos ambientes, desde VD, pouco perturbado, até CB completamente urbanizado, cujas borboletas utilizam flores de jardins para sua alimentação. Água Negra ocupa posição intermediária, por suas características fisionômicas de uma vegetação lenhosa baixa e de média altura, observada nos capões, com abundância de Mirtaceae e Rubiaceae, comuns nesses habitats.

A tabela 1 mostra o número de indivíduos e o número de espécies, por área: em RS e AN foram capturados maior número de indivíduos (271 e 160, respectivamente) e CB e SC o menor (39 e 24, respectivamente). Parides agavus e Parides anchises nephalion foram as espécies mais freqüentes (0,274 e 0,211, respectivamente) e Heraclides anchisiades capys e Protesilaus helios as de menor ocorrência $(0,005$ e 0,004 , respectivamente). As espécies constantes (C) perfazem $33 \%$ e incluem $76,28 \%$ dos indivíduos capturados. As espécies acessórias (A) constituem o grupo maior, com 46,67\%, mas incluem apenas $22,51 \%$ das borboletas. As espécies acidentais (Ac) somam apenas $20 \%$ e constituem somente $1,21 \%$ dos indivíduos (Figura 2).

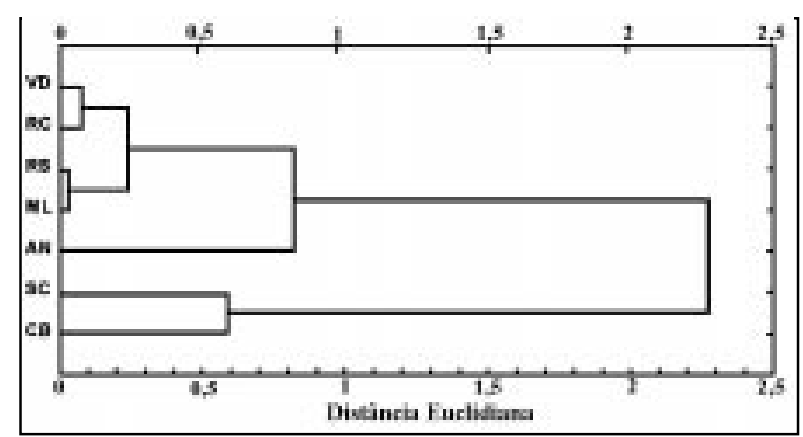

Figura 1 - Análise da similaridade entre as áreas investigadas (VD= Vale do Diabo, $\mathrm{RC}=$ Rincão do Canto, $\mathrm{RS}=$ Rincão do Soturno, $\mathrm{ML}=$ Morro do Link, $\mathrm{AN}=$ ÁguaNegra, $\mathrm{SC}=$ Schöenstatt, $\mathrm{CB}=$ Camobi), com base na cobertura vegetal, usando a Correlação de Pearson. 
Tabela 1 - Espécies, número e freqüência do número de indivíduos capturados por espécie (\%), em sete comunidades de Santa Maria, RS.

\begin{tabular}{|c|c|c|c|c|c|c|c|c|}
\hline ESPÉCIES & RS & $\mathrm{AN}$ & VD & ML & $\mathrm{RC}$ & $\mathrm{CB}$ & $\mathrm{SC}$ & TOTAL \\
\hline Parides agavus (Drury, 1782) & $\begin{array}{c}89 \\
(0,328)\end{array}$ & $\begin{array}{c}27 \\
(0,169)\end{array}$ & $\begin{array}{c}35 \\
(0,321)\end{array}$ & $\begin{array}{c}33 \\
(0,434)\end{array}$ & $\begin{array}{c}18 \\
(0,286)\end{array}$ & - & $\begin{array}{c}1 \\
(0,042)\end{array}$ & $\begin{array}{c}203 \\
(0,274)\end{array}$ \\
\hline Parides anchises nephalion (Godart, 1819) & $\begin{array}{c}57 \\
(0,210)\end{array}$ & $\begin{array}{c}15 \\
(0,937)\end{array}$ & $\begin{array}{c}46 \\
(0,422)\end{array}$ & $\begin{array}{c}17 \\
(0,224)\end{array}$ & $\begin{array}{c}18 \\
(0,286)\end{array}$ & - & $\begin{array}{c}4 \\
(0,167)\end{array}$ & $\begin{array}{c}157 \\
(0,211)\end{array}$ \\
\hline Battus polystictus (Butler,1874) & $\begin{array}{c}25 \\
(0,092)\end{array}$ & $\begin{array}{c}42 \\
(0,262)\end{array}$ & $\begin{array}{c}5 \\
(0,046)\end{array}$ & $\begin{array}{c}4 \\
(0,053)\end{array}$ & $\begin{array}{c}2 \\
(0,032)\end{array}$ & - & $\begin{array}{c}1 \\
(0,042)\end{array}$ & $\begin{array}{c}79 \\
(0,106)\end{array}$ \\
\hline Parides bunichus perrhebus (Boisduval, 1836) & $\begin{array}{c}28 \\
(0,103)\end{array}$ & $\begin{array}{c}31 \\
(0,194)\end{array}$ & $\begin{array}{c}5 \\
(0,046)\end{array}$ & $\begin{array}{c}7 \\
(0,092)\end{array}$ & $\begin{array}{c}1 \\
(0,016)\end{array}$ & $\begin{array}{l}- \\
-\end{array}$ & $\begin{array}{l}- \\
-\end{array}$ & $\begin{array}{c}72 \\
(0,097)\end{array}$ \\
\hline Battus polydamas (Linné,1758) & $\begin{array}{c}20 \\
(0,074)\end{array}$ & $\begin{array}{c}16 \\
(0,100)\end{array}$ & $\begin{array}{c}1 \\
(0,009)\end{array}$ & $\begin{array}{c}3 \\
(0,004)\end{array}$ & - & $\begin{array}{c}10 \\
(0,256)\end{array}$ & $\begin{array}{c}5 \\
(0,208)\end{array}$ & $\begin{array}{c}55 \\
(0,074)\end{array}$ \\
\hline Heraclides astyalus (Godart, 1819) & $\begin{array}{c}18 \\
(0,664)\end{array}$ & $\begin{array}{c}4 \\
(0,025)\end{array}$ & $\begin{array}{c}2 \\
(0,018)\end{array}$ & $\begin{array}{c}3 \\
(0,004)\end{array}$ & $\begin{array}{c}6 \\
(0,095)\end{array}$ & $\begin{array}{c}1 \\
(0,026)\end{array}$ & $\begin{array}{c}6 \\
(0,250)\end{array}$ & $\begin{array}{c}40 \\
(0,054)\end{array}$ \\
\hline Heraclides hectorides (Esper, 1794) & $\begin{array}{c}14 \\
(0,517)\end{array}$ & $\begin{array}{c}6 \\
(0,037)\end{array}$ & $\begin{array}{c}5 \\
(0,046)\end{array}$ & $\begin{array}{c}3 \\
(0,004)\end{array}$ & $\begin{array}{c}1 \\
(0,016\end{array}$ & - & $\begin{array}{c}1 \\
(0,042)\end{array}$ & $\begin{array}{c}30 \\
(0,040)\end{array}$ \\
\hline Mimoides lysithous eupatorion (Hoppfer, 1866) & $\begin{array}{c}8 \\
(0,295)\end{array}$ & $\begin{array}{c}8 \\
(0,050)\end{array}$ & $\begin{array}{c}3 \\
(0,028)\end{array}$ & - & $\begin{array}{c}5 \\
(0,079)\end{array}$ & - & - & $\begin{array}{c}24 \\
(0,032)\end{array}$ \\
\hline Pterourus scamander (Boisduval, 1836) & - & - & - & $\begin{array}{c}1 \\
(0,013)\end{array}$ & $\begin{array}{l}- \\
-\end{array}$ & $\begin{array}{c}20 \\
(0,513)\end{array}$ & $\begin{array}{c}3 \\
(0,125)\end{array}$ & $\begin{array}{c}24 \\
(0,032)\end{array}$ \\
\hline Mimoides lysithous rurik (Eschhscholtz, 1821) & $\begin{array}{c}7 \\
(0,258)\end{array}$ & $\begin{array}{c}2 \\
(0,012)\end{array}$ & $\begin{array}{c}5 \\
(0,046)\end{array}$ & $\begin{array}{c}2 \\
(0,026)\end{array}$ & $\begin{array}{c}5 \\
(0,079)\end{array}$ & - & $\begin{array}{l}- \\
-\end{array}$ & $\begin{array}{c}21 \\
(0,028)\end{array}$ \\
\hline Heraclides thoas brasiliensis (Roths \& Jordan, 1906) & $\begin{array}{c}1 \\
(0,004)\end{array}$ & $\begin{array}{c}3 \\
(0,019)\end{array}$ & - & $\begin{array}{c}3 \\
(0,004)\end{array}$ & $\begin{array}{c}1 \\
(0,016)\end{array}$ & $\begin{array}{c}5 \\
(0,128)\end{array}$ & $\begin{array}{c}2 \\
(0,083)\end{array}$ & $\begin{array}{c}15 \\
(0,020)\end{array}$ \\
\hline Mimoides lysithous lysithous (Hübner,1821) & $\begin{array}{c}3 \\
(0,111)\end{array}$ & $\begin{array}{c}3 \\
(0,019)\end{array}$ & $\begin{array}{c}1 \\
(0,009)\end{array}$ & $\begin{array}{l}- \\
-\end{array}$ & $\begin{array}{c}6 \\
(0,095)\end{array}$ & $\begin{array}{l}- \\
-\end{array}$ & - & $\begin{array}{c}13 \\
(0,017)\end{array}$ \\
\hline Euriades corethrus (Boisduval, 1836) & - & $\begin{array}{c}1 \\
(0,062)\end{array}$ & - & - & $\begin{array}{l}- \\
-\end{array}$ & $\begin{array}{c}3 \\
(0,077)\end{array}$ & - & $\begin{array}{c}4 \\
(0,005)\end{array}$ \\
\hline Heraclides anchisiades capys (Hübner, 1809) & $\begin{array}{c}1 \\
(0,037)\end{array}$ & $\begin{array}{c}1 \\
(0,062)\end{array}$ & $\begin{array}{c}1 \\
(0,009)\end{array}$ & - & - & - & $\begin{array}{c}1 \\
(0,042)\end{array}$ & $\begin{array}{l}4 \\
-\end{array}$ \\
\hline Protesilaus helios (Rothschild \& Jordan, 1906) & - & $\begin{array}{c}1 \\
(0,062)\end{array}$ & - & - & $\begin{array}{l}- \\
-\end{array}$ & - & - & $\begin{array}{c}1 \\
(0,004)\end{array}$ \\
\hline TOTAL & $\begin{array}{c}271 \\
(0,36)\end{array}$ & $\begin{array}{c}160 \\
(0,22)\end{array}$ & $\begin{array}{c}109 \\
(0,15)\end{array}$ & $\begin{array}{c}76 \\
(0,10)\end{array}$ & $\begin{array}{c}63 \\
(0,09)\end{array}$ & $\begin{array}{c}39 \\
(0,05)\end{array}$ & $\begin{array}{c}24 \\
(0,03)\end{array}$ & 742 \\
\hline
\end{tabular}

$\mathrm{RS}=$ Rincão do Soturno; $\mathrm{AN}=$ Água Negra; $\mathrm{VD}=$ Vale do Diabo; $\mathrm{ML}=$ Morro do Link; $\mathrm{RC}=\mathrm{Rincão}$ do $\mathrm{Canto}$; $\mathrm{CB}=\mathrm{Camobi} ; \mathrm{SC}=$ Schöenstatt.

A abundância não é necessariamente um bom indicador da importância das espécies na comunidade (POOLE, 1974). Espécies que ocorrem em muitos locais, com densidades altas, podem ser mais generalistas, em um grupo de espécies com ecologia similar, permitindo uma distribuição ampla (BROWN, 1984). Uma análise do grau de similaridade entre as espécies, com base nas sete áreas (Figura 3), mostra dois grandes grupos: um formado por Parides agavus, Parides anchises nephalion, Parides bunichus perrhebus e Battus polydamas (espécies constantes), Troidiini, ocupando habitats restritos ao interior da mata, exceto Battus polydamas, cosmopolita e comum desde bordas de mata até ambientes urbanos. O segundo grupamento mostra um gradiente, desde Battus polystictus (constante), até Heraclides anchisiades capys (acidental) e incluindo todas as acessórias. Esse grupo se divide em dois menores; um formado por espécies encontradas no interior ou borda de mata (Battus polystictus; Heraclides hectorides; Heraclides astyalus; Mimoides lysithous rurik; Mimoides lysithous eupathorium e Mimoides lysithous lysithous) e outro por espécies associadas a campos abertos ou ambientes urbanos (Pterourus scamander, Heraclides thoas brasiliensis; Heraclides astyalus; Euriades corethrus; Protesilaus helios e Heraclides anchisiades capys), onde estão localizadas as espécies acidentais.

Com base na similaridade e índices de constância, as áreas foram analisados separadamente, agrupando RC e VD, RS e ML e SC com CB. Água Negra foi estudada separadamente. Nos locais com percentual de cobertura vegetal maior, como RC e VD (Figura 4a), Parides agavus e Parides anchises nephalion aparecem juntas no primeiro "cluster", como espécies constantes. No segundo, há uma divisão, onde as subespécies de Mimoides lysithous estão em 


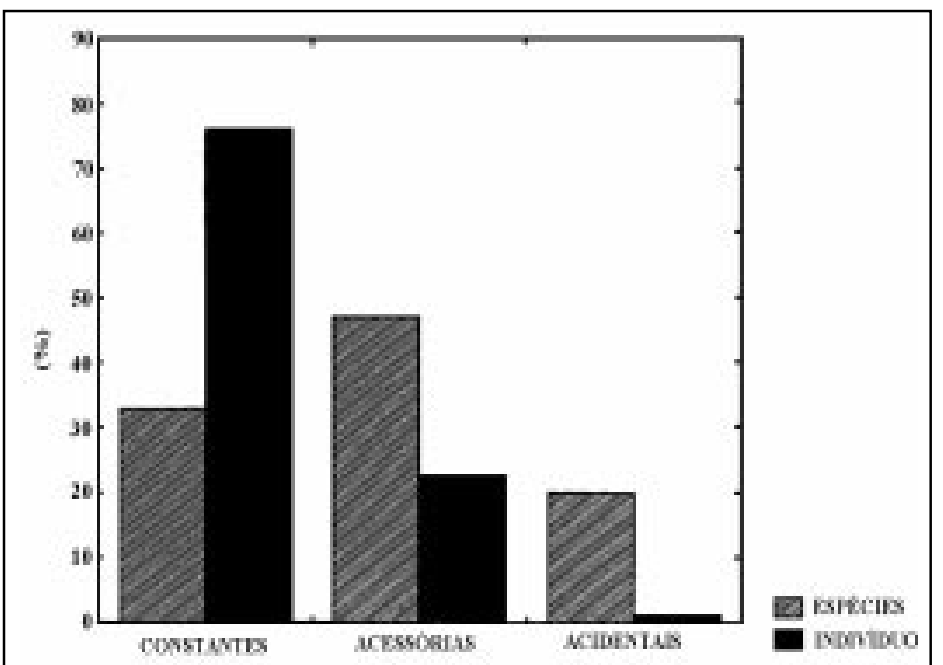

Figura 2 - Proporção de espécies e indivíduos capturados em cada nível de constância.

um e as demais no outro, formado por espécies acidentais e acessórias. Em ML e RS, com cobertura vegetal até $50 \%$, a distribuição (Figura 4 b) é análoga a anterior. Mas, Pterourus scamander, Heraclides thoas brasiliensis e Heraclides anchisiades capys (acidentais), associam-se com as subespécies de Mimoides. Em AN, com vegetação cobrindo até 33\%, a distribuição (Figura 4c) se modifica: Parides agavus, Parides anchises nephalion, Heraclides hectorides e Battus polystictus formam um cluster, todas encontradas em matas. No segundo cluster, existem 2 subgrupos; um formado por Parides bunichus perrhebus, Battus polydamas e Mimoides lysithous eupatorium e outro com as demais. Em CB e SC (Figura 4d), Parides agavus não foi

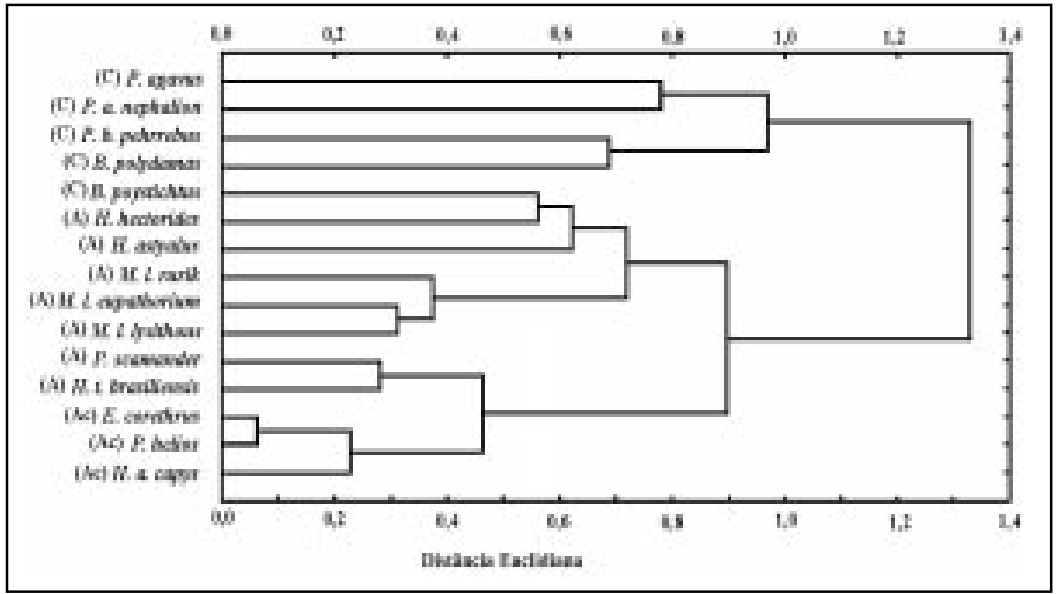

Figura 3 - Fenograma das espécies estudadas ( $\mathrm{C}=$ constantes; $\mathrm{A}=$ acessórias; $\mathrm{Ac}=$ acidentais $)$, com base no número total de indivíduos capturados e no índice de constância de cada espécie. encontrada e Battus polydamas, Heraclides thoas brasiliensis e Pterourus scamander são constantes; observando-se uma inversão, as espécies acessórias em áreas de mata apresentam-se constantes e as constantes como acessórias.

As áreas VD e CB apresentaram os menores índices de diversidade $\left(\mathbf{H}^{\prime}\right)$ (1,596 e 1,246, respectivamente) (Tabela 2) e os valores mais altos em $\mathbf{A N}(2,087)$ e SC (1,972). Uma comparação entre os índices estimados, através de um teste $\mathrm{t}$ (Tabela 3), mostra que AN e CB diferem mais estatisticamente, representando os extremos da distribuição: CB, com uma vegetação pobre associada a plantas ornamentais de jardins e AN com vegetação nativa distribuída pelos capões, determinando uma variabilidade maior. Uma comparação entre as áreas mostra um gradiente na diversidade, em que os valores mais altos estão associados a uma paisagem mais heterogênea, tendo AN e CB seus representantes extremos. A dinâmica das espécies em que ocorrem $\mathbf{A N}$ assemelha-se às previsões realizadas na Teoria do Equilíbrio da Biogeografia de Ilhas (MacARTHUR \& WILSON, 1967): a riqueza de espécies de uma comunidade de herbívoros depende da variação entre as plantas hospedeiras de imaturos e para alimentação de adultos. Dependendo da diversidade de plantas, da similaridade das preferências e performance na utilização das plantas, a estrutura da comunidade será uma conseqüência dessas interações. Borboletas com limites grandes de tolerância podem ampliar sua distribuição mais rapidamente. Os capões atuam como refúgios de inimigos naturais controlando a abundância de espécies e, também, como fonte de indivíduos para colonização da área.

O Índice de Simpson, alternativo ao de ShannonWiener, quando o número de espécies é insuficiente (COLINVAUX, 1993), neste estudo reflete a mesma tendência nos padrões observados. Valores mais elevados para AN e SC podem estar associados ao fato de que habitats mais complexos suportam um número mais elevado de espécies herbívoras, porque 


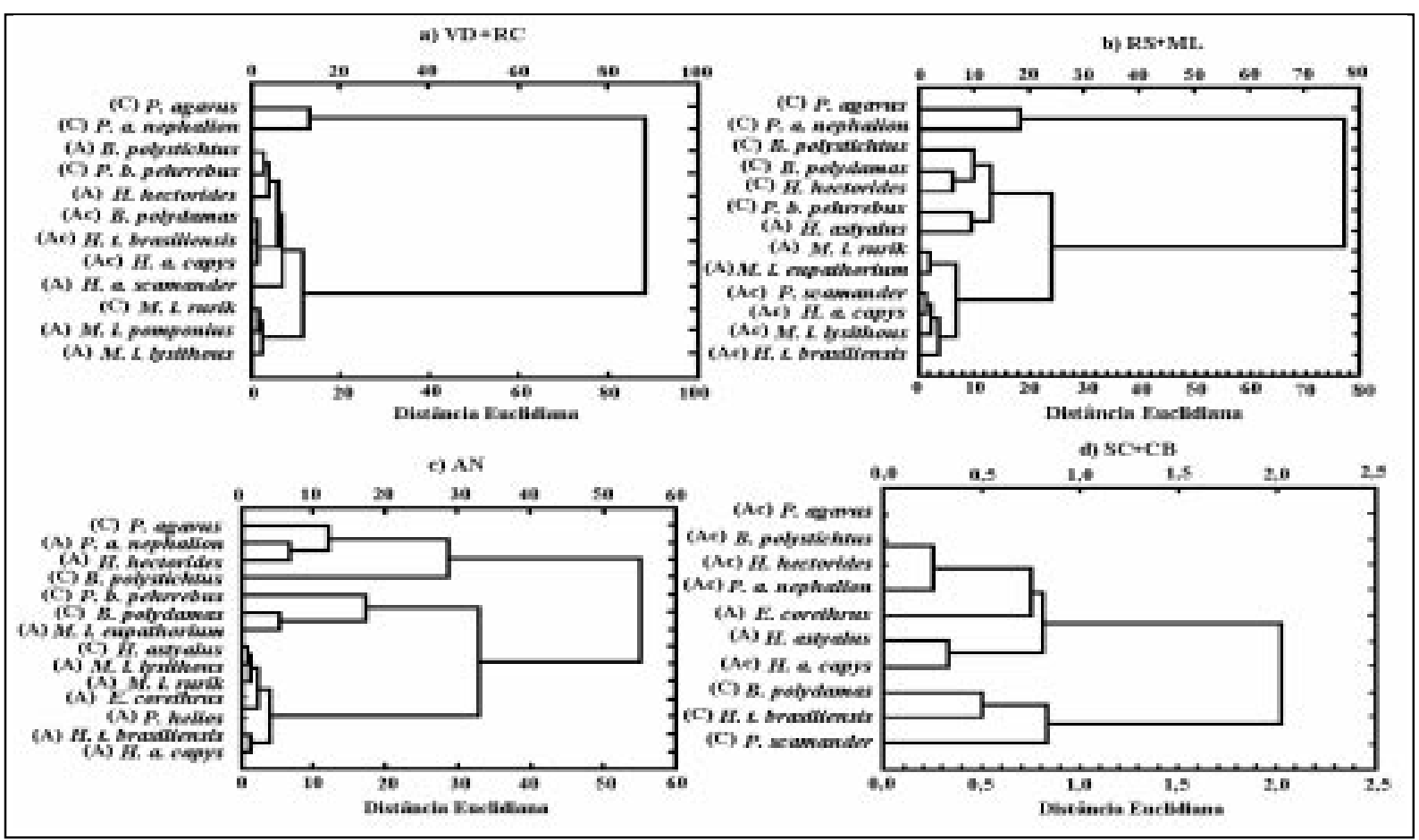

Figura 4 - Fenograma das espécies estudadas $(\mathrm{C}=$ constantes; $\mathrm{A}=$ acessórias; $\mathrm{Ac}=$ acidentais $)$, com base nas localidades investigadas, nas sete comunidades de Santa Maria, RS.

apresentam características que são responsáveis pela diversidade observada nesses locais (PRICE, 1984).

A importância da abundância de espécies (Figura 5), expressa pela riqueza e uniformidade de espécies, mostra que a tendência mais uniforme e, conseqüentemente, com maior diversidade é a de $\mathbf{A N}$, seguida pela de $\mathbf{S C}$, enquanto $\mathbf{C B}$ apresentou a menor. Entretanto, apesar de $\mathbf{A N}$ e $\mathbf{S C}$ apresentarem os maiores índices de diversidade, $\mathbf{S C}$, devido a suas características urbanas, apresentou a menor densidade. Desse modo, a alta diversidade apresentada, provavelmente, é devido a altas taxas de migração de indivíduos e espécies que apresentam

Tabela 2 - Equitabilidade, índices de Simpson e de Shannon-Wiener e variância das estimativas dos índices de Shannon-Wiener para as sete comunidades de Santa Maria, RS.

\begin{tabular}{llllllll}
\hline & AN & SC & RS & RC & ML & VD & CB \\
\hline N & 160 & 24 & 271 & 63 & 76 & 109 & 39 \\
NE & 14 & 9 & 12 & 10 & 10 & 11 & 5 \\
Simpson & 0,841 & 0,837 & 0,814 & 0,804 & 0,743 & 0,704 & 0,648 \\
S. Wiener (H') & 2,087 & 1,972 & 1,964 & 1,874 & 1,737 & 1,596 & 1,246 \\
Equitabilidade & 1,00 & 0,945 & 0,941 & 0,898 & 0,832 & 0,765 & 0,595 \\
Var (H') & 0,0048 & 0,0159 & 0,0023 & 0,0115 & 0,0141 & 0,0112 & 0,0140 \\
\hline
\end{tabular}

$\mathrm{AN}=$ Água Negra; $\mathrm{SC}=$ Schöenstatt; $\mathrm{RS}=$ Rincão do Soturno; $\mathrm{RC}=$ Rincão do Canto; $\mathrm{ML}=$ Morro do Link; $\mathrm{VD}=$ Vale do Diabo; $\mathrm{CB}=$ Camobi.

$\mathrm{N}=$ número de indivíduos capturados; $\mathrm{NE}=$ número de espécies observadas;

H'= índice de Shannon-Wiener; Var (H')= variância da estimativa de H'. um comportamento oportunista. Tal fato pode ser inferido com Parides agavus, acidental em SC e CB, espécie encontrada em locais de floresta e de baixa dispersão, embora seus movimentos dentro das áreas de vida sejam intensos.

\section{CONCLUSÕES}

A utilização de modelos que estudam a relação de abundância entre espécies auxilia a resumir analogias e criar hipóteses eurísticas sobre as interações entre as espécies de uma comunidade. Os resultados obtidos, neste estudo, mostram que a estrutura e a cobertura da vegetação desempenham papel importante na ocupação e distribuição de Papilionidae nas áreas investigadas, permitindo uma subdivisão dos ambientes ocupados. A análise separada das áreas indica, também, que os mecanismos que influenciam a diversidade em escalas mais amplas são diferentes dos mecanismos que influenciam a diversidade em escalas menores.

Espécies com ampla distribuição, como Parides agavus e Parides anchises nephalion, demonstram que o nível de constância pode estar associado com padrões na estrutura vegetal dos ambientes que ocupam. 
Tabela 3 - Comparação dos índices de diversidade H' (índice de Shannon-Wiener), através do teste t de Student nas sete comunidades de Santa Maria, RS.

\begin{tabular}{|c|c|c|c|c|c|c|}
\hline & AN & $\mathrm{SC}$ & $\mathrm{RS}$ & $\mathrm{RC}$ & ML & VD \\
\hline $\mathrm{SC}$ & 0,799 (ns) & - & - & - & - & - \\
\hline $\mathrm{RS}$ & $1,411(\mathrm{~ns})$ & $0,058(\mathrm{~ns})$ & & & & \\
\hline $\mathrm{RC}$ & $1,668\left(^{*}\right)$ & $0,592(\mathrm{~ns})$ & $0,753(\mathrm{~ns})$ & & & \\
\hline ML & $2,546(* *)$ & $1,530(\mathrm{~ns})$ & $1,746(*)$ & $0,856(\mathrm{~ns})$ & & \\
\hline VD & $3,882(* *)$ & $2,284(*)$ & $3,110(* *)$ & $1,845(*)$ & $0,886(\mathrm{~ns})$ & \\
\hline $\mathrm{CB}$ & $6,134(* *)$ & $4,199(* *)$ & $5,539(* *)$ & $3,933(* *)$ & $2,929(* *)$ & $2,205(*)$ \\
\hline
\end{tabular}

$\mathrm{AN}=$ Água Negra; $\mathrm{SC}=$ Schöenstatt; RS= Rincão do Soturno; RC= Rincão do Canto; $\mathrm{ML}=$ Morro do Link; VD= Vale do Diabo; $\mathrm{CB}=$ Camobi $* \mathrm{p}<0.05 ; * * \mathrm{p}<0.01 ; \mathrm{ns}=$ não significante.

A dominância de Papilionidae, negativamente correlacionada com a abundância e diversidade da vegetação, é conseqüência da interação entre a heterogeneidade real do ambiente e as habilidades dos organismos de perceber e deslocarem-se pelo ambiente. Alguns ambientes, como os capões ou locais urbanos com vegetação para fins paisagísticos, podem permitir que as atividades nessas áreas possam ser favorecidas, minimizando os efeitos da heterogeneidade ambiental onde pode estar disponível uma concentração de alimentos. As borboletas podem ser capazes de converter ambientes heterogêneos em homogêneos por alimentarem-se e viverem em diferentes tipos de locais, que dependem do grau de heterogeneidade espacial, podendo serem mais efetivas em evitar a competição exclusiva entre os organismos. Estudos mais amplos, e empregando outros grupos de insetos, poderão ser de grande utilidade para aumentar o entendimento das bases genéticas, fisiológicas e demográficas dos espaços ocupados pelos organismos e o papel que a diversidade da vegetação exerce sobre a diversidade e abundância das espécies.

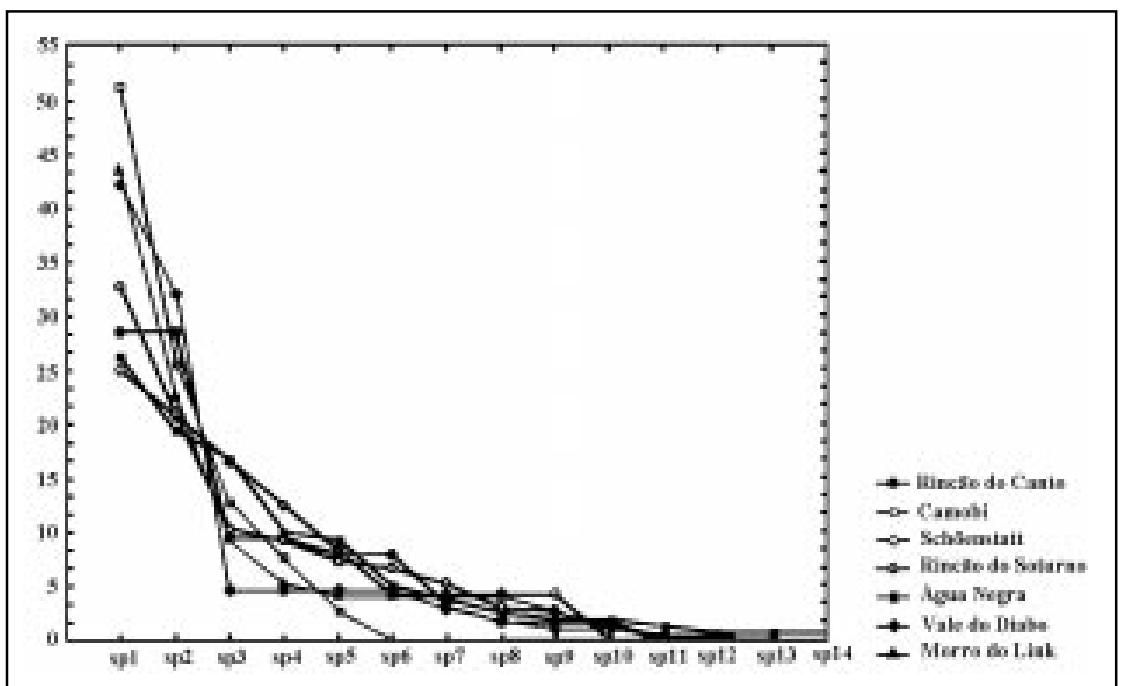

Figura 5 - Curva de importância das espécies estudadas, com base nos indivíduos capturados nas sete comunidades de Santa Maria, RS

\section{REFERÊNCIAS BIBLIOGRÁFICAS}

ACKERY, P.R., VANE-WRIGHT, R.I. Milkweed butterflies: their cladistic and biology. Ithaca, New York : Comstock, 1984. 425p.

BODENHEIMER, F.S. Precis d'écologie animale. Paris: Payot, 1955. 315p.

BROWN, V.K. Secondary Sucession: insect-plant relationships. Bioscience, v.34, p.710716,1984 .

COLINVAUX, P. Ecology. New York: John Willey \& Sons, 1993. 688p.

HEPNNER, J.B. Faunal regions and the diversity of lepidoptera. Tropical Tepidoptera, Gainesville FL, v.2, n.1, p.1-85, 1991.

HUSTON, A.H. Biological diversity: the coexistence of species on changing landscapes. Cambridge : Cambridge University, 1995. 681p.

KLEIN, R.M. Aspectos fitofisionômicos da floresta estacional na fralda da Serra Geral (RS). In: CONGRESSO NACIONAL DE BOTÂNICA, 1983, Porto Alegre. Anais... Porto Alegre : Sociedade de Botânica do Brasil, 1983. p.192.

MACARTHUR, R.H., WILSON, E.D. The theory of island biogeography. New Jersey : Princeton University, 1967. 203 p.

POOLE, R.W. Introduction to quantitative ecology. Tokyo: Mc Graw-Hill, 1974. 532p

PRICE, T.D. The evolution of sexual size dimorphism in Darwin's finches selection. American Naturalist, Chicago, v.123, p.500-518, 1984

SUGIHARA, G. Minimal community structure: an explanation of species abundance patterns. American Naturalist, Chicago, v.116, p.770-787, 1980.

TYLER, H.A., BROWN Jr., K.S., WILSON, K.H. Swallowtail butterflies of the Americas: a study in biological, biosystematic and conservation. Gainesville: Scientific Publishers, 1994. 376p.

WARD, J.H. Hierarchical grouping to optimize an objective function. Journal of the American Statistical Association Alexandria US, v.58, p.236, 1963.

WHITTAKER, R.H. Vegetation of the Siskiyou Mountains, Oregon and California. Ecological Monographs, Arizona, v.30, p.279-338, 1960 .

WHITTAKER, R.H Gradient analysis of vegetation. Biological Reviews Cambridge, v.42, p.207264, 1967.

WILSON, M.V., MOHLER, C.L. Measuring compositional Change along gradients. Vegetatio, Dordrecht, v.54, p.129-141, 1983. 